\title{
Effectiveness of Immunoglobulin Replacement Therapy on Clinical Outcome in Patients with Primary Antibody Deficiencies: Results from a Multicenter Prospective Cohort Study
}

\author{
Isabella Quinti - Annarosa Soresina • Andrea Guerra • \\ Roberto Rondelli • Giuseppe Spadaro • Carlo Agostini • \\ Cinzia Milito • Amelia Chiara Trombetta • \\ Marcella Visentini • Helene Martini • \\ Alessandro Plebani • Massimo Fiorilli • \\ IPINet Investigators
}

Received: 19 November 2010 / Accepted: 4 February 2011 /Published online: 2 March 2011

(C) Springer Science+Business Media, LLC 2011

\begin{abstract}
A 5-years multicenter prospective study on 201 patients with common variable immunodeficiencies and 101 patients with X-linked agammaglobulinemia over a cumulative follow-up period of 1,365 patient-years was conducted to identify prognostic markers and risk factors for associated clinical co-morbidities, the effects of longterm immunoglobulin treatment and the $\mathrm{IgG}$ trough level to be maintained over time required to minimise infection risk.
\end{abstract}

Italian Primary Immunodeficiencies Network (IPINet): Bari (D De Mattia, B Martire, F Cardinale, G Ranieri, F Silvestri), Bologna (M Masi), Bologna Centro Operativo AIEOP (A Pession), Cagliari ( $\mathrm{F}$ Cossu), Catanzaro (E Anastasio), Catania (G Schillirò), Firenze (A Matucci, A Vultaggio, M Aricò), Milano (MC Pietrogrande, RM Delle Piane, C Panisi, G Cambiaghi), Napoli (C Pignata), Padova (MC Putti), Palermo (A Trizzino), Parma (P Bertolini), Pisa (R Consolini), Roma (AG Ugazio, M Duse, M Iacobini, V Moschese, C Cancrini, A Finocchi, AM Pesce, M Cagliuso, V Conti, G Granata, M Mitrevski), Salerno (F Cecere), Torino (PA Tovo, S Martino, D Montin), Varese (L Nespoli, M Marinoni, FP Pellegrini) and Verona (GA Cazzola).

Electronic supplementary material The online version of this article (doi:10.1007/s10875-011-9511-0) contains supplementary material, which is available to authorized users.

I. Quinti $(\bowtie) \cdot$ A. Guerra $\cdot$ C. Milito $\cdot$ A. C. Trombetta

M. Visentini $\cdot$ H. Martini $\cdot$ M. Fiorilli

Department of Clinical Immunology,

Sapienza University of Rome,

Viale dell'Università 37,

00185 Rome, Italy

e-mail: isabella.quinti@uniroma1.it

\author{
A. Soresina $\cdot$ A. Plebani \\ Department of Pediatrics, University of Brescia, \\ Brescia, Italy
}

Overall, $21 \%$ of the patients with common variable immunodeficiencies and $24 \%$ of patients with X-linked agammaglobulinemia remained infection free during the study. A reduction of pneumonia episodes has been observed after initiation of Ig replacement. During the observation time, pneumonia incidence remained low and constant over time. Patients with pneumonia did not have significant lower IgG trough levels than patients without

\footnotetext{
R. Rondelli

Department of Onco-haematology, University of Bologna, Bologna, Italy

G. Spadaro

Department of Clinical Immunology, University of Naples, Naples, Italy

C. Agostini

Department of Clinical Immunology, University of Padua, Padua, Italy
} 
pneumonia, with the exception of patients whose $\operatorname{IgG}$ trough levels were persistently $<400 \mathrm{mg} / \mathrm{dL}$. In X-linked agammaglobulinemia, the only co-morbidity risk factor identified for pneumonia by the final multivariable model was the presence of bronchiectasis. In common variable immunodeficiencies, our data allowed us to identify a clinical phenotype characterised by a high pneumonia risk: patients with low IgG and IgA levels at diagnosis; patients who had IgA level $<7 \mathrm{mg} / \mathrm{dL}$ and who had bronchiectasis. The effect of therapy with immunoglobulins at replacement dosage for non-infectious co-morbidities (autoimmunity, lymphocytic hyperplasia and enteropathy) remains to be established. A unique general protective trough $\operatorname{IgG}$ level in antibody deficiency patients will remain undefined because of the major role played by the progression of lung disease in X-linked agammaglobulinemia and in a subset of patients with common variable immunodeficiencies.

Keywords Immunoglobulin replacement · common variable immunodeficiency $\cdot \mathrm{X}$-linked agammaglobulinemia $\cdot$ bronchiectasis $\cdot \operatorname{IgA} \cdot$ effectiveness

\section{Introduction}

Survival of patients with primary antibody deficiencies has increased due to improved detection of infectious diseases, availability of Ig replacement therapy and antimicrobial agents. IgG replacement therapy has been stated as beneficial [1], although the optimal IgG trough level to be maintained over time in order to minimise infectious risk is not established. Consequently, there is a wide variation in treatment practices [2-7]. A summary of guidelines on the management of primary antibody deficiency with replacement therapy suggested achieving trough $\mathrm{IgG}$ serum levels of at least $5 \mathrm{~g} / \mathrm{L}$ and ideally of $6.5-10 \mathrm{~g} / \mathrm{L}$ [8]. More recently, a study on a cohort of common variable immunodeficiencies (CVIDs) and X-linked agammaglobulinemia (XLA) English patients [9] and a meta-analysis on the impact of trough IgG on pneumonia incidence [10] provided evidence that patients might remain infection free and the pneumonia risk can be progressively reduced by higher trough IgG levels in order to reach a "biologic" level that is unique for each patient [11]. Severe infections are the main cause of morbidity and mortality in XLA patients [12]. Clinical phenotypes of CVIDs patients are more heterogeneous and patients are susceptible to a wide range of co-morbidities other than infections [13]. Therefore, the suggested protective high trough $\mathrm{IgG}$ level might not be considered a general goal and only large prospective multi-centre studies might help to identify CVIDs subgroups of patients at high infection risk. We have carried out a prospective study with the aim to identify prognostic markers and risk factors for associated clinical complications in a cohort of 302 patients with primary antibody deficiencies over a cumulative follow-up period of 1,365 patient-years.

\section{Methods}

Patients and Study Design In year 2001, a total of 302 patients diagnosed according to the ESID/PAGID criteria for XLA and CVIDs [14] and registered in the Italian Primary Immunodeficiency Network (IPINET) database have been included in this prospective study. One hundred and one patients had a definitive diagnosis of XLA based on $<2 \%$ of peripheral B cells and mutations of the Btk gene; 201 patients had a diagnosis of CVIDs based on a marked decrease (at least 2 standard deviation (SD) below the mean for age) in serum $\operatorname{IgG}$ and $\operatorname{IgA}$, onset age $>2$ years, poor response to vaccines and exclusion of other causes of hypogammaglobulinemia. Data has been prospectively collected for 5 years according to the study protocol and inserted in an internet-based patient database on a yearly basis. Detailed information with personal data, pedigree, date of diagnosis, immunological data and clinical manifestations, route and dosage of immunoglobulin replacement from the time of enrolment were collected in a structured questionnaire filled by one physician per centre on a yearly basis. All data were processed in a database and sent to the Interuniversity Computing Center (CINECA) responsible for processing and analysing the data. For each patient, full blood counts, lymphocyte subsets, chemistries, serum immunoglobulin levels were performed four times per year. Chest and sinus computerised tomography (CT) scans were performed every 4 years, gastrointestinal endoscopy with biopsy every 2 years or when indicated (Supplemental Table I). Retrospective data related to diagnosis was also available (study design: Supplemental Figure 1). All patients were on intravenous or subcutaneous immunoglobulins according to the national guidelines (www.aieop.org). All participants provided written informed consent. The study protocol was approved by the Institutional Review Board at Università Sapienza of Rome.

Measurements Disease-associated clinical co-morbidities such as X-ray defined pneumonia, acute sinusitis, meningitis, sepsis, bronchiectasis confirmed by $\mathrm{CT}$, histologicailly defined chronic lymphatic hyperplasia, chronic diarrhoea, splenomegaly confirmed by ultrasound, autoimmunity were recorded. The diagnosis of chronic lung disease was based on clinical findings, pulmonary function tests and chest thinsection CT examinations. The diagnosis of chronic sinusitis was based on clinical findings and confirmed with the CT scan of sinonasal cavities, performed in a period free of 
acute symptoms [15]. The diagnosis of pneumonia was made according to guidelines from The Infectious Disease Society of America [16].

Statistical Analysis Parametric and non-parametric data is presented as mean $\pm \mathrm{SD}$ or range, as indicated. For comparison of clinical and biomarker changes between groups, the Student $T$ test and Mann-Whitney test were used for parametric or non-parametric datasets. Multiple proportional hazards analysis was performed to determine independent predictors of any clinical condition studied, after selecting relevant significant variables by univariate analysis. Analyses were performed separately for each outcome. The risk for each clinical condition was calculated by Cox proportional hazards models with the given clinical condition as the dichotomous outcome. The time-to-event variable was the time from enrolment in the prospective study to the first episode of that clinical condition. Subjects who did not develop that condition were censored at the time of their last followup or death. Because of the many clinical and immunological variables we considered in the univariate analyses (Supplemental Table I), some of which were highly correlated; we evaluated each of these variables as a potential confounder. In the final multivariable models, we included those factors known to be of clinical importance or that seemed to confound the relationship between risk factors and the outcome variable. To detect a possible level of protection of different Ig isotypes we used a receiver operating characteristic (ROC) curve, that is a graphical plot of the sensitivity vs. (1-specificity) for a binary classifier system as its discrimination threshold is varied. We used SAS, JMP8 version (SAS Institute, Cary, North Carolina), for all statistical analyses. Results were expressed as hazard ratios (HR) with $95 \% \mathrm{CIs}$ and $p$ values. A $p$ value of $<0.05$ was taken as the threshold of statistical significance.

\section{Results}

\section{Patients Characteristics}

The prospective study included 201 CVIDs (97 females, 104 males) and 101 XLA patients. CVIDs patients were followed up for a cumulative period of 778 patient-years (mean time 3.8 years follow-up per patient) and XLA for 587 patient-years (mean time 5.8 years). At diagnosis, the mean age was $28.7 \pm 18.4$ years for CVIDs (range, 3-68) and $4.9 \pm 6.2$ years for XLA (range, 16 days -40.9 years). At the end of the study, mean age was $40.5 \pm 18.9$ years for CVIDs and $21 \pm 5$ years for XLA.
At diagnosis immunoglobulins levels (Supplemental Tables II and III) were: IgG $252.1 \pm 133.2 \mathrm{mg} / \mathrm{dL}$ (CVIDs) and $139.6 \pm 103 \mathrm{mg} / \mathrm{dL}$ (XLA); IgA $18.6 \pm 24.3 \mathrm{mg} / \mathrm{dL}$ (CVIDs) and $7 \pm 6.1 \mathrm{mg} / \mathrm{dL}$ (XLA); IgM $32.6 \pm 47.4 \mathrm{mg} / \mathrm{dL}$ (CVIDs) and $13.8 \pm 12.9 \mathrm{mg} / \mathrm{dL}$ (XLA). In CVIDs, IgG, IgA, IgM levels did not differ between females and males.

The delta $\operatorname{IgG}$ (mean IgG trough levels at the end of the study period minus the $\operatorname{IgG}$ level at diagnosis) was $415.2 \pm$ $197.8 \mathrm{mg} / \mathrm{dL}$ in patients with CVID and $577.9 \pm 208.7 \mathrm{mg} /$ dL in patients with XLA (Supplemental Tables II and III). The trough IgG levels at the time of the study were slightly correlated with the Ig replacement dose in XLA patients without chronic diarrhoea $(r=0.12 ; p=0.047)$, and in CVID patients without chronic diarrhoea and without chronic lymphatic hyperplasia $(r=0.22 ; p<0.0001$; data not shown).

Is There a Direct Relationship Between Ig Level and Reduced Infections?

Overall, $79 \%$ of the patients with CVIDs and $76 \%$ of patients with XLA had major infectious complications (at least one episode of pneumonia and/or acute sinusitis or had a condition of chronic lung disease with bronchiectasis). As shown in Table I, CVIDs patients who experienced infections had lower IgA $(17 \pm 26 \mathrm{mg} / \mathrm{dL})$ and $\operatorname{IgM}(34 \pm$ $77 \mathrm{mg} / \mathrm{dL}$ ) serum levels than patients who did not have infections (IgA: $34 \pm 36 \mathrm{mg} / \mathrm{dL}$; IgM: $42 \pm 43 \mathrm{mg} / \mathrm{dL}$ ) $(p<0.001)$. IgG trough levels and the monthly mean $\operatorname{IgG}$ dose administered were stable over time in most of our patients. However, in some of them, in particular those with enteropathy (manuscript in preparation), increasing the Ig dosage did not result in an adequate increase in IgG trough levels. In other patients raising the IgG trough level did not prevent episodes of pneumonia. The monthly mean Ig dose administered was lower in CVIDs patients without infections $(350 \pm 116 \mathrm{mg} / \mathrm{kg} / \mathrm{month})$ in comparison to patients who had infections $(398 \pm 167 \mathrm{mg} /$ $\mathrm{kg} /$ month). XLA patients without or with infections received higher doses than CVID patients (594 \pm 176 and $608 \pm 273 \mathrm{mg} / \mathrm{kg} / \mathrm{month}$, respectively; Table I).

\section{Acute Infections}

Pneumonia A significant reduction in the prevalence of pneumonia, the main XLA- and CVIDs-associated acute infection [17-21], has been observed after initiation of immunoglobulin replacement therapy in XLA (from 38.5\% to $25.7 \%, p=0.037$ ) and CVIDs patients (from $39.4 \%$ to $22.3 \%, p<0.0001)$. During the prospective study the incidence of pneumonia remained relatively constant both in XLA (range 0.03-0.11 episodes/patient/year) and in CVIDs (range 0.06-0.10 episodes patient-year). It has been previously suggested that the total number of infectious 
Table I Mean IgG, IgA, IgM $( \pm \mathrm{SD})(\mathrm{mg} / \mathrm{dL})$ serum levels and mean $( \pm \mathrm{SD})$ monthly IgG dosage administered (milligrams per kilogram per month) in "infectious" and "non infectious" CVID and XLA patients and in patients with none, one or more than one episode of pneumonia during the 5 years of the prospective study

\begin{tabular}{|c|c|c|c|c|c|}
\hline & "Non infectious" & "Infectious" & No pneumonia & 1 episode of pneumonia & $>1$ episodes of pneumonia \\
\hline \multicolumn{6}{|l|}{ CVID } \\
\hline Number of patients & 42 & 159 & 160 & 25 & 16 \\
\hline $\operatorname{IgG}$ & $622 \pm 179$ & $667 \pm 176$ & $652 \pm 159$ & $725 \pm 211$ & $594 \pm 226^{*}$ \\
\hline $\operatorname{IgA}$ & $34 \pm 36$ & $17 \pm 26^{*}$ & $23 \pm 31$ & $14 \pm 24 *$ & $6 \pm 8^{*}$ \\
\hline $\operatorname{IgM}$ & $42 \pm 43$ & $34 \pm 77^{*}$ & $34 \pm 61$ & $48 \pm 120$ & $23 \pm 35^{*}$ \\
\hline $\begin{array}{l}\text { Monthly Ig replacement dose } \\
\text { XLA }\end{array}$ & $350 \pm 116$ & $398 \pm 167$ & $375 \pm 156$ & $463 \pm 193$ & $415 \pm 88$ \\
\hline Number of patients & 24 & 77 & 76 & 13 & 12 \\
\hline $\mathrm{IgG}$ & $768 \pm 140$ & $758 \pm 202$ & $749 \pm 167$ & $747 \pm 158$ & $753 \pm 172$ \\
\hline $\operatorname{IgA}$ & $6 \pm 6$ & $5.6 \pm 9$ & $6 \pm 9$ & $3 \pm 2$ & $4 \pm 6$ \\
\hline $\operatorname{IgM}$ & $8 \pm 7$ & $6.2 \pm 7$ & $6 \pm 6$ & $6 \pm 5$ & $4 \pm 5$ \\
\hline Monthly Ig replacement dose & $594 \pm 176$ & $608 \pm 273$ & $589 \pm 190$ & $550 \pm 110$ & $712 \pm 278$ \\
\hline
\end{tabular}

$* p<0.0001$

episodes per patient is lower in patients who receive highdose immunoglobulin replacement therapy $[4,5,7,9,10]$. Overall, in the 5 years of observation, episodes of pneumonia developed in 41/201 CVIDs patients and in 25/101 XLA patients (Fig. 1). Patients with pneumonia did not have significant lower IgG trough levels than patients without pneumonia. When patients were grouped into IgG trough level intervals, only the group with persistently $\mathrm{IgG}$ trough $<400 \mathrm{mg} / \mathrm{dL}$ had an increased risk to have pneumonia (HR 5.1; $p<0.0009$; Table II). In CVID, lower IgG levels at diagnosis ( $<250 \mathrm{vs}>250 \mathrm{mg} / \mathrm{dL}$; HR, $1.2 ; p=0.04$ ) and higher delta $\mathrm{IgG}$ (a difference $>430 \mathrm{mg} /$ $\mathrm{dL}$ between the IgG trough level at the end of the study period and the IgG level at diagnosis; HR, $1.5 ; p=0.006$ ) were significantly associated with the subsequent occurrence of pneumonia (IgG at diagnosis: $209.9 \pm 123.5 \mathrm{mg} /$ $\mathrm{dL}$, pneumonia group; $263.4 \pm 133.8 \mathrm{mg} / \mathrm{dL}$, no pneumonia group; and delta IgG: $493.7 \pm 220.4 \mathrm{mg} / \mathrm{dL}$, pneumonia group; $394.1 \pm 186.6 \mathrm{mg} / \mathrm{dL}$, no pneumonia group).

CVIDs patients with more than one episode of pneumonia (16/41) during the 5 years of observation had lower mean values of $\operatorname{IgA}(6 \pm 8 \mathrm{mg} / \mathrm{dL})$, and $\operatorname{IgM}(23 \pm 35 \mathrm{mg} / \mathrm{dL})$ in comparison with patients who had only one episode (IgA $14 \pm 24$, and $\operatorname{IgM} 48 \pm 120 \mathrm{mg} / \mathrm{dL} ; p<000.1)$ and with patients without pneumonia (IgA $23 \pm 31$, and $\operatorname{IgM~} 34 \pm$ $61 \mathrm{mg} / \mathrm{dL} ; p<0.0001$; Table I).

Since pre-existing co-morbidity factors may obscure the effects of $\mathrm{Ig}$ replacement therapy, we performed a multivariate analysis. The major co-morbidity risk factor identified for pneumonia was the presence of bronchieactasis (HR, 3; $p=0.007$ for CVIDs; HR, 3.52; $p=0.004$ for XLA; Table II).
Within the CVIDs cohort (Table II), a significant increase in the risk of pneumonia (HR, 5.1; $p=0.0009$ ) was found only in those patients who did not reach an $\mathrm{IgG}$ trough level $>400 \mathrm{mg} / \mathrm{dL}$. Using ROC curves we did not identify a statistically valid cut-off value of $\mathrm{IgG}$, when we only analysed data collected during the prospective study where IgG levels were higher. When data collected at diagnosis before $\mathrm{Ig}$ replacement were included in the analysis, a pneumonia cutoff value of $408 \mathrm{mg} / \mathrm{dL}$ was found, albeit with a low sensitivity (62\%) and a low specificity $(75 \%)$. This also showed that even so, not everyone gets pneumonia and other risk factors such as bronchiectasis and low IgA played a major role. Within the XLA cohort, episodes of pneumonia occurred in patients over a wide range of $\mathrm{IgG}$ trough levels (Fig. 1), with a slight increase in the hazard risk for patients who did not reach an $\mathrm{IgG}$ trough levels $>500 \mathrm{mg} / \mathrm{dL} \quad(p=0.049$; Table II). It has been recently suggested that IgG levels should be kept at high levels [10] in order to prevent pneumonia. However, in our XLA cohort, only 1 episode of pneumonia was observed when IgG trough levels were kept higher than $1,000 \mathrm{mg} / \mathrm{dL}$. This might suggest that very high IgG trough levels might be protective, even if we couldn't statistically confirm this hypothesis due to the low number of observations that falls into this trough IgG interval.

In CVID patients, serum IgA levels were lower in patients who had pneumonia $(11.3 \pm 18.6 \mathrm{mg} / \mathrm{dL})$ than in patients who never developed pneumonia $(24.1 \pm 31.8 \mathrm{mg} / \mathrm{dL})(p=0.02$; Fig. 2). Using ROC analysis, the statistically chosen cut-off value for IgA levels was $7 \mathrm{mg} / \mathrm{dL}$, with a sensitivity of $84 \%$ and a specificity of $48 \%$. In patients with $\operatorname{IgA}$ lower than $7 \mathrm{mg} / \mathrm{dL}$ there was an increase risk of pneumonia (HR, 2.6; $p=0.03$ ), independently of other risk factors. 


\section{A IgG}

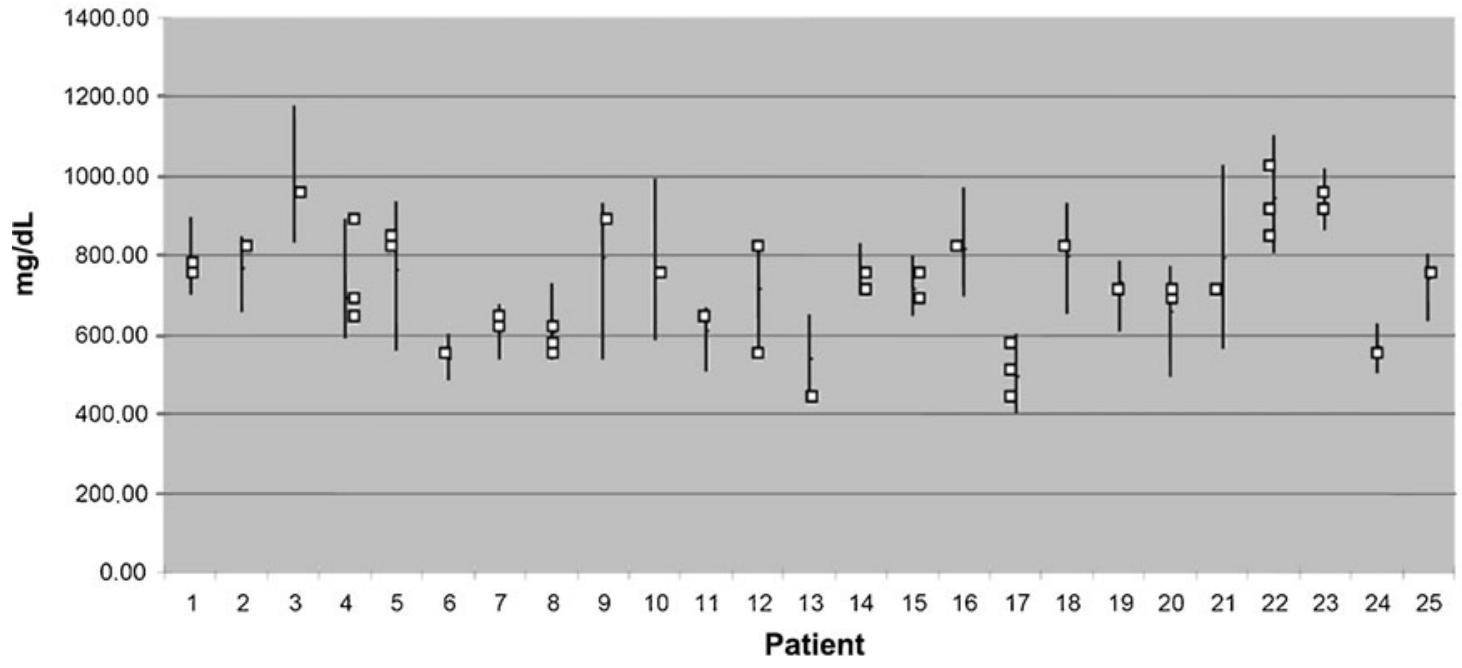

\section{B IgG}

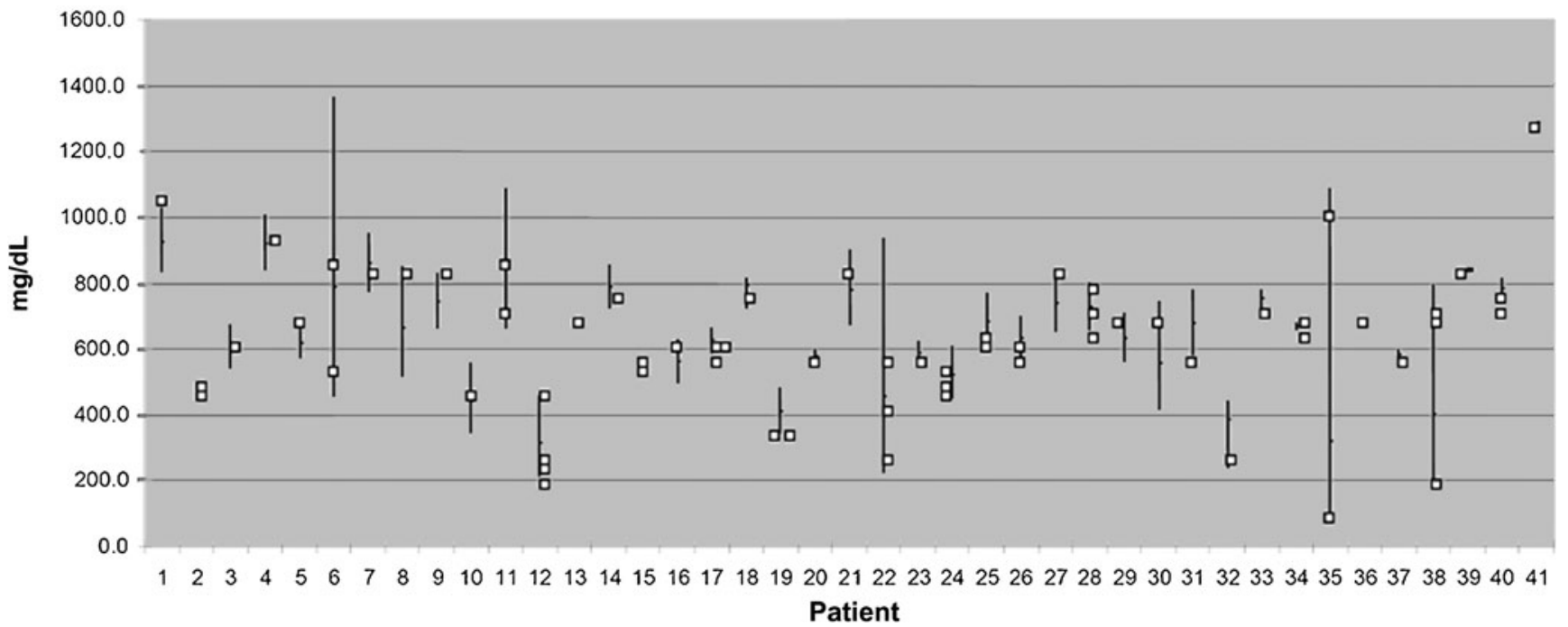

Fig. 1 IgG level (milligrams per deciliter, mg/dL; white squares) at the time of each distinct episode of pneumonia in the 25 XLA patients (panel $A$ ) and in 41 CVIDs patients (panel $B$ ) who had pneumonias during the prospective study. Black lines indicate the range with geometric means of IgG trough level (milligrams per deciliter) of each patient during the study time
Acute Sinusitis Data on acute sinusitis were only available for CVIDs patients. As expected, the multivariate analysis identified as risk factors for acute sinusitis only chronic sinusitis (HR 11.4, $p<0.0001)$ and bronchiectasis (HR 1.6, $p=0.02)$.

Invasive Bacterial Infections A drastic reduction in the incidence of invasive infections, bacterial sepsis and meningitis, has been observed after initiation of replacement therapy in both XLA from $6 \%$ to $2.4 \%$ (0.003 episodes patient-year) and CVIDs patients from $16.6 \%$ to $2.5 \%$ (0.0007 episodes patient-year).

\section{Chronic Conditions}

Bronchiectasis Over a 5-year observation period, bronchiectasis prevalence increased from $47.3 \%$ at the time of enrolment to $53.7 \%$ at the last year of follow-up in CVIDs patients and from $33 \%$ to $39 \%$ in XLA patients. No significant difference was observed in the mean $\operatorname{IgG}$ trough levels between patients who developed $(648 \pm 171 \mathrm{mg} / \mathrm{dL})$ or did not develop $(650 \pm 147 \mathrm{mg} / \mathrm{dL})$ bronchiectasis.

In CVIDs, by univariate analysis IgA and IgM levels, age, age of disease onset, pneumonia, acute and chronic 
Table II Risk factors and hazard risks (HR) for CVIDs and XLA associated co-morbidities identified by multiple regression analysis

\begin{tabular}{llcc}
\hline Clinical condition & Risk factor & HR & $p$ \\
\hline CVIDs & & & \\
Pneumonia & Basal IgG, $<250 \mathrm{vs}>250 \mathrm{mg} / \mathrm{dL}$ & 0.04 \\
& Delta IgG, $>430 \mathrm{vs}<430 \mathrm{mg} / \mathrm{dL}$ & 1.2 & 0.006 \\
& IgG trough, $<400 \mathrm{mg} / \mathrm{dL}$ vs $>400 \mathrm{mg} / \mathrm{dL}$ & 1.5 & 0.0009 \\
& IgA, $<7 \mathrm{mg} / \mathrm{dL}$ vs $>7 \mathrm{mg} / \mathrm{dL}$ & 5.1 & 0.03 \\
& Bronchiectasis & 2.6 & 0.007 \\
Acute sinusitis & Chronic sinusitis & 3 & $<0.0001$ \\
& Bronchiectasis & 11.4 & 0.02 \\
Bronchiectasis & IgA, $<7 \mathrm{mg} / \mathrm{dL}$ vs $>7 \mathrm{mg} / \mathrm{dL}$ & 1.6 & 0.04 \\
& Age & 2.4 & 0.0007 \\
Chronic sinusitis & IgA, $<7 \mathrm{mg} / \mathrm{dL}$ vs $>7 \mathrm{mg} / \mathrm{dL}$ & $1.5^{\mathrm{a}}$ & 0.02 \\
& Acute sinusitis & 3.7 & $<0.0001$ \\
Splenomegaly & Age & 11.5 & 0.01 \\
& IgA, $<7 \mathrm{mg} / \mathrm{dL}$ vs $>7 \mathrm{mg} / \mathrm{dL}$ & $1.2^{\mathrm{a}}$ & 0.02 \\
XLA & Bronchiectasis & 2.2 & 0.0003 \\
Pneumonia & & 2.6 & 0.005 \\
Bronchiectasis & Chronic lung disease & & 0.049 \\
Chronic sinusitis & IgG trough, $<500 \mathrm{mg} / \mathrm{dL}$ vs $>500 \mathrm{mg} / \mathrm{dL}$ & 3.5 & 1.1 \\
\hline
\end{tabular}

Multiple proportional hazards analysis was performed to determine independent predictors of any clinical condition studied, after selecting relevant significant variables by univariate analysis

In the final multivariable models, those factors known to be of clinical importance or that seemed to confound the relationship between risk factors and the outcome variable were evaluated

${ }^{a} 10$-years intervals) length of time of disease was not a risk factor for bronchiectasis.

Splenomegaly In CVIDs, a spleen enlargement is a common finding in patients with granulomatous disease and/or chronic lymphatic hyperplasia [22]. However, its prevalence is much higher than that related to these CVIDsassociated conditions [17, 19]. In our CVIDs cohort, the prevalence of patients with splenomegaly (longitudinal diameter $>12 \mathrm{~cm}$, identified by ultrasound) increased during the observation period from $31 \%$ to $42.3 \%$. Low IgA and IgM levels, age, age at onset, bronchiectasis, acute and chronic sinusitis, pneumonia, chronic lymphatic hyperplasia and autoimmunity were found to be associated with splenomegaly by univariate analysis. By multiple regres-
Fig. 2 Mean IgA levels (mg/dl) \pm SD in CVID patients with or without pneumonias (panel $A$ ). IgA levels (mg/dl) in CVID patients with (black) or without pneumonias (grey). Patients are grouped in percentile intervals according to their IgG trough level. Each dot represents a single year of follow-up (panel B)
A

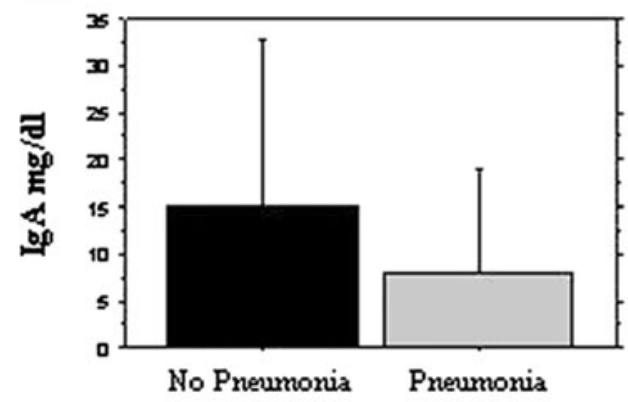

B

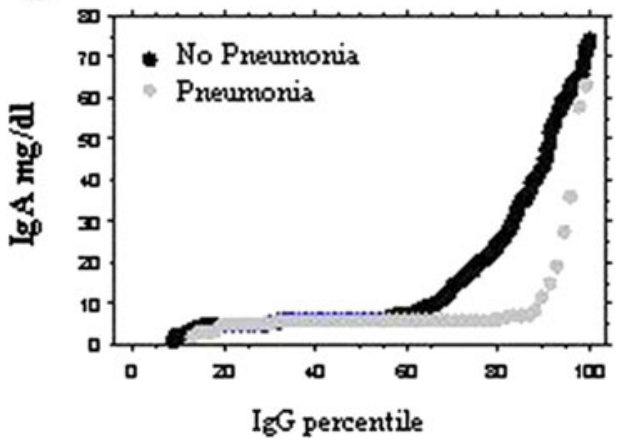


sion bronchiectasis (HR 2.6, $p=0.0003$ ) and low IgA levels $(p=0.004)$ were independent risk factors for splenomegaly (Table II). By ROC analysis, the statistically chosen cut-off value for IgA levels was $7 \mathrm{mg} / \mathrm{dl}$, (sensitivity $70.2 \%$; specificity $61.1 \%$ ). Patients with $\operatorname{IgA}<7 \mathrm{mg} / \mathrm{dL}$ had an increased risk of splenomegaly (HR 2.2, $p=0.02$ ).

Chronic Sinusitis In the CVIDs cohort, the prevalence of patients with chronic sinusitis, increased over time from $45.3 \%$ at enrolment to $52.2 \%$ in CVID and from $50 \%$ to $51 \%$ in XLA. In CVIDs, its occurrence was associated with IgA serum level of $<7 \mathrm{mg} / \mathrm{dL}$ (HR 3.7, $p=0.02$ ), and with acute sinusitis (HR 11.5, $p<0.0001$; Table II). In XLA, chronic sinusitis was highly associated with bronchiectasis (HR, 3.8; $p=0.005$ ).

\section{Discussion}

The events that define the pathogenesis of an infection depend on a large range of variables related to specific infecting organisms and to the overall immunological state of the host. In primary antibody deficiencies, where the defect is the inability to produce an effective antibody response to pathogens, only $\operatorname{IgG}$ antibodies might be replaced by intravenous or subcutaneous administration. Evidence of immunoglobulin replacement efficacy has been demonstrated in small trials comparing high- and low-dose immunoglobulin doses and from several observational studies $[1-8,15,17-19$, 23]. Recently, a study by Chapel et al. [9] and a meta-analysis [10] on the impact of trough IgG on pneumonia incidence provided evidence that pneumonia risk can be progressively reduced by higher trough IgG levels up to a "biologic" level [11]. Replacement doses greater than $600 \mathrm{mg} / \mathrm{kg} / \mathrm{month}$ and trough levels $>1,000 \mathrm{mg} / \mathrm{dL}$ have been suggested. In this context, what do we learn from this "real life" observation of patients treated with lower doses, a common practice in many immunological centres?

Clinical phenotypes of CVIDs and XLA, the most common symptomatic primary antibody deficiencies requiring a longlife Ig substitution, are quite variable even within one disease or syndrome. Therefore, the suggested protective trough IgG level of $1,000 \mathrm{mg} / \mathrm{dl}$ might not be considered a general goal and only large prospective multi-centre studies might help to identify subgroups of patients at high infection risk. This prospective study identified prognostic markers and risk factors for associated clinical co-morbidities in a large cohort of patients with primary antibody deficiencies over a long cumulative follow-up period. After starting on replacement therapy a reduction in the incidence of severe acute infections was observed. Thereafter, this incidence remained low and constant over time. The trough IgG levels measured at the time of each episode of pneumonia were extremely variable and were not lower in those patients who experienced one or more pneumonia episodes. In XLA, the only co-morbidity risk factor identified for pneumonia was the presence of bronchiectasis and bronchiectasis was the major risk factor for pneumonia. This means that in order to modify the XLA clinical course our efforts should be directed to the prevention of pneumonia. The same observation was only partially true for CVIDs in which bronchiectasis was the major risk factor for pneumonia, while age and low IgA levels but not pneumonia was the major risk factors for bronchiectasis. Moreover, our data allowed identifying a clinical phenotype characterised by a high pneumonia risk: patients who had low $\operatorname{IgG}$ and IgA levels at diagnosis; patients who had IgA level $<7 \mathrm{mg} / \mathrm{dL}$ and who had bronchiectasis.

International guidelines on immunoglobulin replacement suggest a similar monthly $\operatorname{IgG}$ dose to be administered in XLA and CVIDs [1]. In our study, the mean IgG dose administered to XLA patients was higher than that administered to CVIDs. This choice might reflect a greater apprehension of physicians regarding the risk of severe infections in XLA. A similar variability was recently found by the Chapel's group [9]. In both XLA and CVIDs, we could not identify a unique IgG trough level and an optimal IgG replacement dose to prevent pneumonia, because of the major role played by the progression of lung disease as risk factor. However, when data from the prospective study were cumulated with retrospective data we found that in CVIDs, the hazard risk for pneumonia increased in those patients who did not reach an $\operatorname{IgG}$ trough level $>400 \mathrm{mg} / \mathrm{dL}$. This figure was not evident in the recent paper from Chapel et al. [9] as they did not have any IgG trough levels that were this low. This data does not indicate that the protective trough $\operatorname{IgG}$ level is $400 \mathrm{mg} / \mathrm{dL}$, but it remarks a higher risk to develop pneumonia at low IgG levels. Our results prompted us to support the still controversial recommendation to start Ig replacement treatment, even before the development of a severe infection, when IgG are that low.

Low $\operatorname{IgG}$ and $\operatorname{IgA}$ at diagnosis and very low IgA level at the prospective study, reflecting a severe impaired isotype switching process, were major independent risk factors for lung complications, confirming that loss of function of memory B cells represents a major cause of CVIDs-infectious-associated clinical conditions, as demonstrated in CVIDs with bronchiestasis [24-26]. A classification of CVIDs based on memory B cell frequency has been proposed, but how this biomarker relates to CVIDs clinical phenotypes remains uncertain [27, 28]. While infections are the main cause of morbidity in all XLA patients, the same might not be true for all CVIDs. In fact, non infectious complications (autoimmunity, lymphocityc hyperplasia and enteropathy) are unique clinical phenotypes in subgroup of CVIDs patients who 
might not develop infections and might have an unclear benefit from Ig replacement [13]. The effect of therapy with immunoglobulins at replacement dosage for noninfectious co-morbidities (autoimmunity, lymphocytic hyperplasia and enteropathy) remains to be established.

We conclude that a unique general protective trough IgG level in antibody deficiency patients will remain undefined because of the major role played by the progression of lung disease. Aside from Ig replacement, which greatly contributed to the reduction of invasive infections, a strategy to reduce lung damage should be approached. Our still ongoing study represents call for further prospective evaluations that should analyse the effectiveness of Ig replacement on the different clinical and immunological forms of antibody deficiencies.

Acknowledgments We wish to thank all the patients who participated to this study as well as their families, and the Jeffrey Modell Foundation. This work was supported by grant of the European Commission, HEALTH-F2-2008-201549

Conflict of Interests The authors declare no competing financial interests.

\section{References}

1. Orange JS, JS HEM, et al. Use of intravenous immunoglobulin in human disease: a review of evidence by members of the Primary Immunodeficiency Committee of the American Academy of Allergy, Asthma and Immunology. J Allergy Clin Immunol. 2006;117:S525-53.

2. Cunningham-Rundles C, Siegal FP, Smithwick EM, et al. Efficacy of intravenous immunoglobulin in primary humoral immunodeficiency disease. Ann Intern Med. 1984;101:435-9.

3. Busse PJ, Razvi S, Cunningham-Rundles C. Efficacy of intravenous immunoglobulin in the prevention of pneumonia in patients with common variable immunodeficiency. J Allergy Clin Immunol. 2002;109:1001-4.

4. Quartier P, Debre M, De Blic J, et al. Early and prolonged intravenous immunoglobulin replacement therapy in childhood agammaglobulinemia: a retrospective survey of 31 patients. J Pediatr. 1999;134:589-96.

5. Roifman CM, Levison H, Gelfand EW. High-dose versus lowdose intravenous immunoglobulin in hypogammaglobulinaemia and chronic lung disease. Lancet. 1987;1:1075-7.

6. Chapel HM, Spickett GP, Ericson D, Engl W, Eibl MM, Bjorkander J. The comparison of the efficacy and safety of intravenous versus subcutaneous immunoglobulin replacement therapy. J Clin Immunol. 2000;20:94-100.

7. Eijkhout HW, van Der Meer JW, Kallenberg CG, et al. The effect of two different dosages of intravenous immunoglobulin on the incidence of recurrent infections in patients with primary hypogammaglobulinemia. A randomized, double-blind, multicenter crossover trial. Ann Intern Med. 2001;135:165-74.

8. Roifman CM, Berger M, Notarangelo LD. Management of primary antibody deficiency with replacement therapy: summary of guidelines. Immunol Allergy Clin N Am. 2008;28:875-6.

9. Lucas M, Lee M, Lortan J, Lopez-Granados E, Misbah S, Chapel $\mathrm{H}$. Infection outcomes in patients with common variable immu- nodeficiency disorders: relationship to immunoglobulin therapy over 22 years. J Allergy Clin Immunol. 2010;125:1354-60.

10. Orange JS, Grossman WJ, Navickis RJ, Wilkes MM. Impact of trough IgG on pneumonia incidence in primary immunodeficiency: a meta-analysis of clinical studies. Clin Immunol. 2010;137:21-30.

11. Bonagura VR, Marchlewski R, Cox A, Rosenthal DW. Biologic IgG level in primary immunodeficiency disease: the $\operatorname{IgG}$ level that protects against recurrent infection. J Allergy Clin Immunol. 2008;122(1):210-2.

12. Plebani A, Soresina A, Rondelli R, et al. Clinical, immunological, and molecular analysis in a large cohort of patients with X-linked agammaglobulinemia: an Italian multicenter study. Clin Immunol. 2002;104:221-30.

13. Chapel H, Lucas M, Lee M, et al. Common variable immunodeficiency disorders: division into distinct clinical phenotypes. Blood. 2008;112:277-86.

14. Conley ME, Notarangelo LD, Etzioni A. Diagnostic criteria for primary immunodeficiencies. Representing PAGID (Pan-American Group for Immunodeficiency) and ESID (European Society for Immunodeficiencies). Clin Immunol. 1999;93:190-7.

15. Mafee MF, Tran BH, Chapa AR. Imaging of rhinosinusitis and its complications: plain film, CT, and MRI. Clin Rev Allergy Immunol. 2006;30:165-86.

16. Bartlett JG, Dowell SF, Mandell LA, File Jr TM, Musher DM, Fine MJ. Practice guidelines for the management of communityacquired pneumonia in adults. Infectious Diseases Society of America. Clin Infect Dis. 2000;31(2):347-82.

17. Cunningham-Rundles $\mathrm{C}$, Bodian C. Common variable immunodeficiency: clinical and immunological features of 248 patients. Clin Immunol. 1999;92:34-48.

18. Kainulainen L, Varpula M, Liippo K, Svedstrom E, Nikoskelainen J, Ruuskanen O. Pulmonary abnormalities in patients with primary hypogammaglobulinemia. J Allergy Clin Immunol. 1999;104:1031-6.

19. Quinti I, Soresina A, Spadaro G, et al. Long-term follow-up and outcome of a large cohort of patients with common variable immunodeficiency. J Clin Immunol. 2007;27:308-16.

20. Sweinberg SK, Wodell RA, Grodofsky MP, Greene JM, Conley ME. Retrospective analysis of the incidence of pulmonary disease in hypogammaglobulinemia. J Allergy Clin Immunol. 1991;88:96-104.

21. Wood P, Stanworth S, Burton J, et al. Recognition, clinical diagnosis and management of patients with primary antibody deficiencies: a systematic review. Clin Exp Immunol. 2007;149:410-23.

22. Bates CA, Ellison MC, Lynch DA, Cool CD, Brown KK, Routes JM. Granulomatous-lymphocytic lung disease shortens survival in common variable immunodeficiency. J Allergy Clin Immunol. 2004; $114: 415-21$.

23. Ochs HD, Fischer SH, Wedgwood RJ, et al. Comparison of high-dose and low-dose intravenous immunoglobulin therapy in patients with primary immunodeficiency diseases. Am J Med. 1984;76:78-82.

24. Carsetti R, Rosado MM, Donnanno S, et al. The loss of IgM memory $B$ cells correlates with clinical disease in common variable immunodeficiency. J Allergy Clin Immunol. 2005;115:412-7.

25. Alachkar H, Taubenheim N, Haeney MR, Durandy A, Arkwright PD. Memory switched B cell percentage and not serum immunoglobulin concentration is associated with clinical complications in children and adults with specific antibody deficiency and common variable immunodeficiency. Clin Immunol. 2006;120:310-8.

26. Sanchez-Ramon S, Radigan L, Yu JE, Bard S, Cunningham-Rundles C. Memory B cells in common variable immunodeficiency: clinical associations and sex differences. Clin Immunol. 2008;128:314-21.

27. Park MA, Li JT, Hagan JB, Maddox DE, Abraham RS. Common variable immunodeficiency: a new look at an old disease. Lancet. 2008;372:489-502.

28. Wehr C, Kivioja T, Schmitt C, et al. The EUROclass trial: defining subgroups in common variable immunodeficiency. Blood. 2008;111:77-85. 